Supporting Information 


\title{
Challenging Dogmas: Hydrogen Bond Revisited
}

\author{
Maxim Tafipolsky \\ June 10, 2016
}

\begin{abstract}
Institut für Physikalische und Theoretische Chemie, Universität Würzburg, Campus Hubland Nord, Emil-Fischer-Strasse 42, D-97074 Würzburg, Germany
\end{abstract}


Table S1: Cartesian coordinates of the linear and bifurcated water dimers (in Angstrom).

\begin{tabular}{cccc}
\hline Atom & $\mathrm{x}$ & $\mathrm{y}$ & $\mathrm{z}$ \\
\hline \multicolumn{4}{c}{ linear } \\
O1 & 0.000000 & 0.000000 & 0.000000 \\
$\mathrm{H} 2$ & 0.971600 & 0.000000 & 0.000000 \\
$\mathrm{H} 3$ & -0.246390 & 0.000000 & -0.939840 \\
$\mathrm{O} 4$ & -0.881200 & 0.000000 & -2.774100 \\
$\mathrm{H} 5$ & -1.411830 & 0.769220 & -3.040080 \\
$\mathrm{H} 6$ & -1.411830 & -0.769220 & -3.040080 \\
\multicolumn{4}{c}{ bifurcated } \\
O1 & 0.000000 & 0.000000 & 0.000000 \\
H2 & 0.000000 & 0.000000 & 0.971600 \\
H3 & 0.939840 & 0.000000 & -0.246387 \\
O4 & -2.411929 & 0.000000 & -1.866842 \\
H5 & -1.941645 & -0.768338 & -1.502841 \\
H6 & -1.943450 & 0.770099 & -1.504238 \\
\hline
\end{tabular}



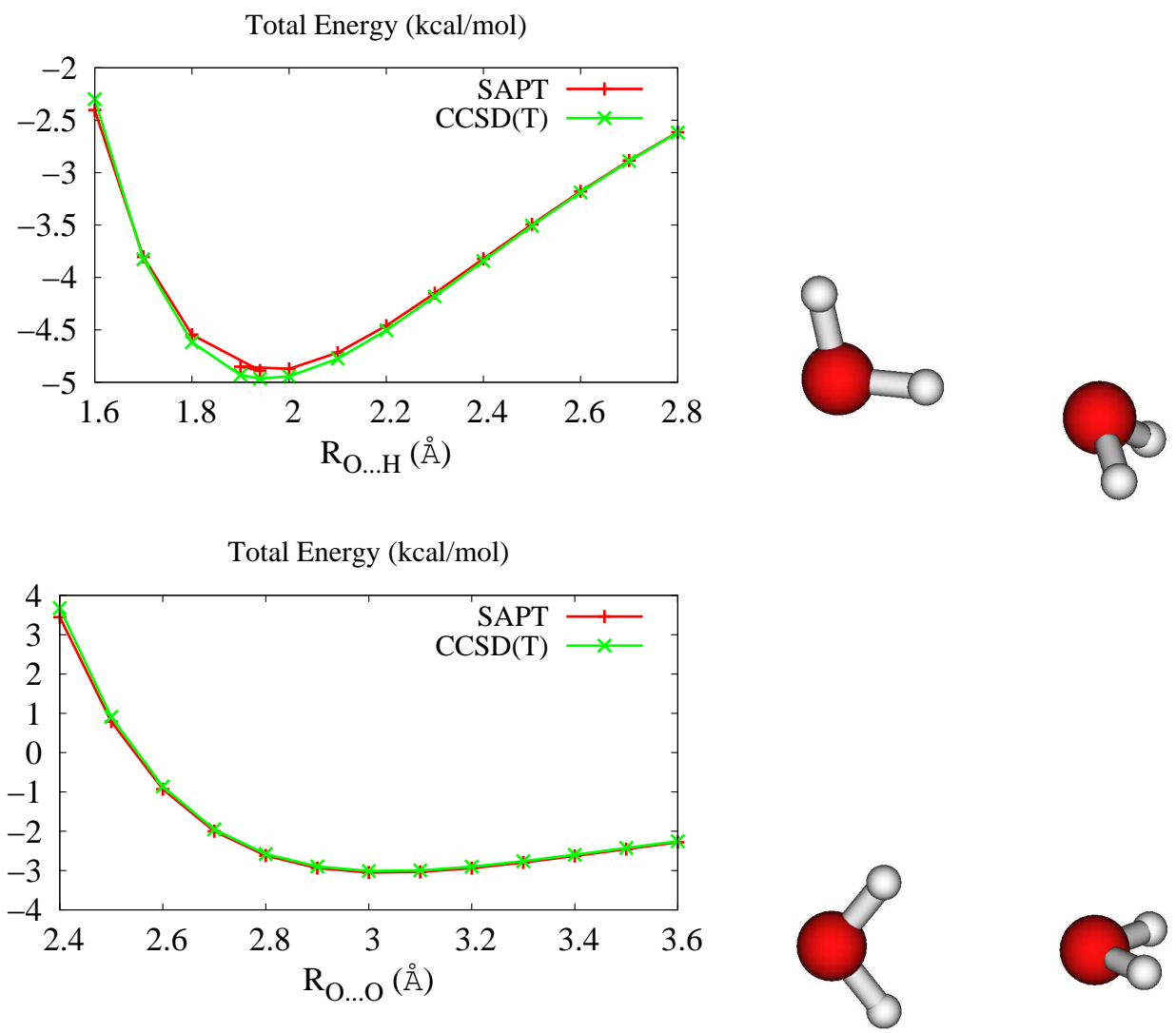

Figure S1: Total interaction energies from DFT-SAPT/aug-cc-pVQZ and CCSD(T)/aug-cc-pVQZ as a function of the H...O (linear dimer, top) and O...O (bifurcated dimer, bottom) separation (in $\mathrm{kcal} / \mathrm{mol}$ ). 

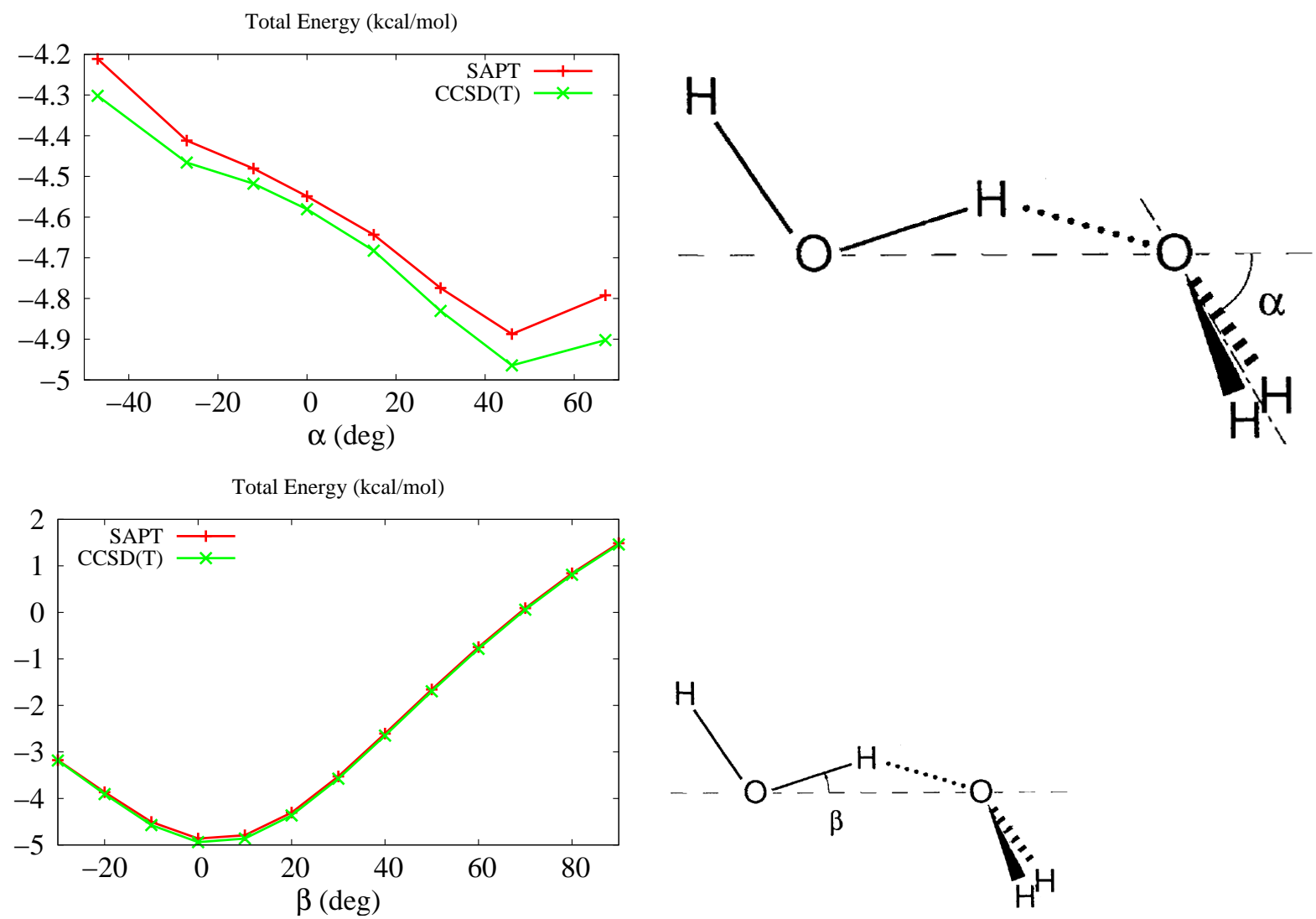

Figure S2: Angular dependence of the total interaction energies from DFTSAPT/aug-cc-pVQZ and CCSD(T)/aug-cc-pVQZ (in kcal/mol). 


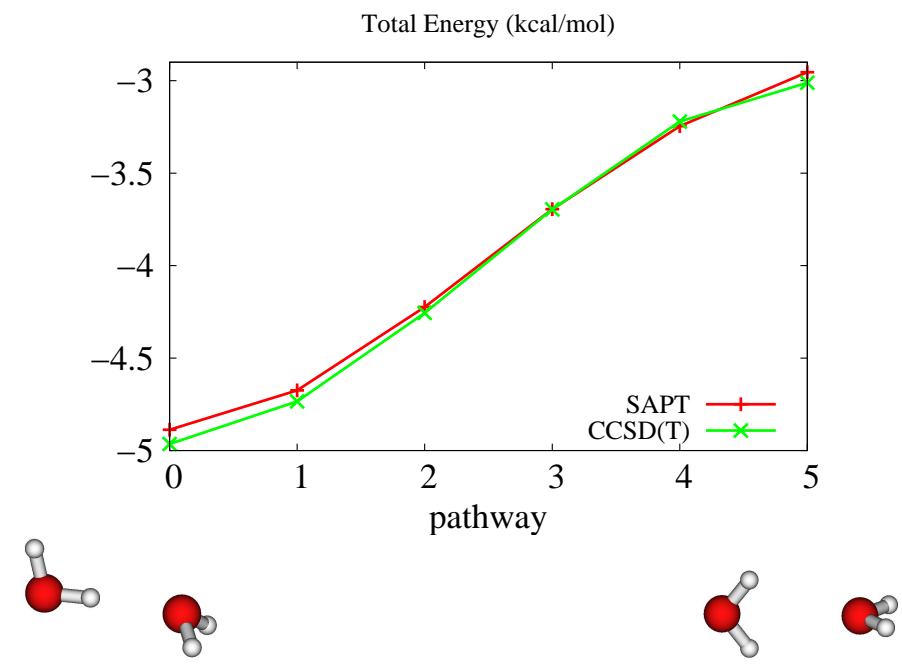

Figure S3: Total interaction energies (in $\mathrm{kcal} / \mathrm{mol}$ ) from DFT-SAPT/aug-ccpVQZ and CCSD(T)/aug-cc-pVQZ along the pathway from the linear configuration (marked as "0") to the bifurcated one (marked as " 5 "). 
Table S2: DFT-SAPT energy decomposition analysis for 10 stationary points of the water dimer (in $\mathrm{kcal} / \mathrm{mol}$ )*

\begin{tabular}{cccccc}
\hline dimer** & $E_{\text {elst }}$ & $E_{\text {exch }}$ & $E_{\text {disp }}$ & $E_{\text {ind }}$ & $E_{\text {tot }} * * *$ \\
\hline 1 (linear) & -7.9183 & 7.8115 & -2.3933 & -2.2508 & $-4.75(-4.85)$ \\
2 & -6.6823 & 6.4804 & -2.1780 & -1.9083 & $-4.29(-4.39)$ \\
3 & -6.4836 & 6.1967 & -2.1350 & -1.8485 & $-4.27(-4.37)$ \\
4 & -6.5964 & 5.9922 & -2.3264 & -1.1076 & $-4.04(-4.11)$ \\
5 & -5.6740 & 5.0139 & -2.2057 & -0.9610 & $-3.83(-3.91)$ \\
6 (cyclic) & -5.3610 & 4.6392 & -2.1685 & -0.9067 & $-3.80(-3.90)$ \\
7 & -4.8573 & 4.1420 & -1.8861 & -0.5423 & $-3.14(-3.17)$ \\
8 & -1.4805 & 1.2005 & -0.8983 & -0.1355 & $-1.31(-1.28)$ \\
9 (bifurcated) & -4.7400 & 4.2164 & -1.8149 & -0.6521 & $-2.99(-3.02)$ \\
10 & -2.7087 & 2.1541 & -1.2492 & -0.3834 & $-2.19(-2.22)$ \\
\hline
\end{tabular}

* DFT-SAPT: LPBE0AC/aug-cc-pVQZ (see main text for details).

** The structures are taken from Reinhardt, P. and Piquemal, J. -P.

Int. J. Quant. Chem. 2009, 109, 3259 originally proposed by van Duijneveldt-van de Rijdt, J. G. C. M.; Mooij, W. T. M.; van Duijneveldt, F. B. Phys. Chem. Chem. Phys. 2003, 5, 1169.

These are based on rigid monomers using experimental geometries with $\mathrm{r}(\mathrm{O}-\mathrm{H})=0.9572 \AA$ and $<\mathrm{H}-\mathrm{O}-\mathrm{H}=104.52 \mathrm{deg}$.

*** SAPT(Hybrid) in parentheses from Reinhardt, P. and Piquemal, J. -P.

Int. J. Quant. Chem. 2009, 109, 3259.

Table S3: Angular variables along the pathway (in degrees).

\begin{tabular}{ccc}
\hline dimer & $\alpha$ & $\beta$ \\
\hline 0 (linear) & 46 & 3 \\
1 & 36 & 7 \\
2 & 26 & 17 \\
3 & 16 & 27 \\
4 & 6 & 37 \\
5 (bifurcated) & 0 & 52 \\
\hline
\end{tabular}



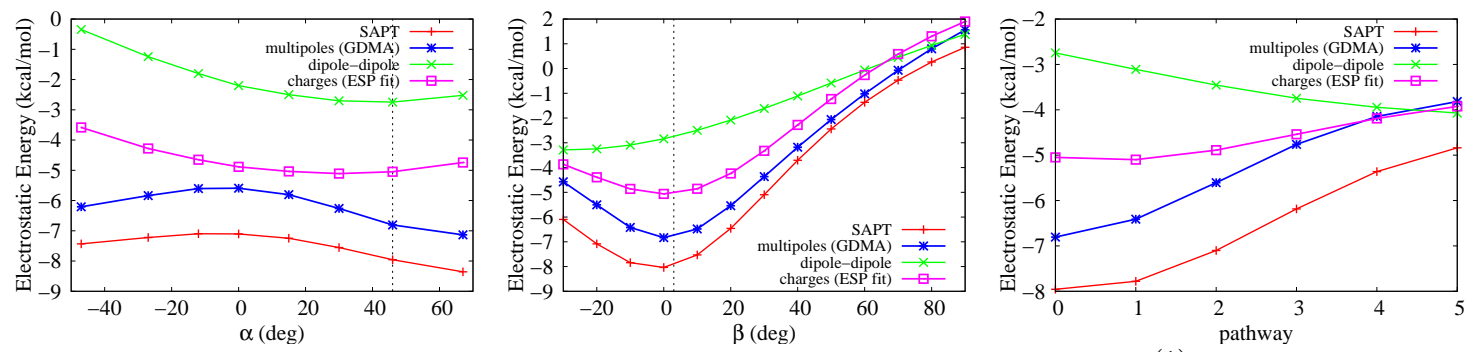

Figure S4: Angular dependence of the electrostatic energy from SAPT, $E_{\text {elst }}^{(1)}$, (in $\mathrm{kcal} / \mathrm{mol}$ ) against various approximate models for the water dimer with the O...O distance fixed at $2.91 \AA$. See Fig. S2 for the definition of the angles $\alpha$ and $\beta$. The pathway defines the transition from the linear configuration (marked as " 0 ") to the bifurcated one (marked as "5"). The vertical broken line marks the approximate equilibrium angle. The atom-centered multipoles calculated with the GDMA program (Stone, A. J. J. Chem. Theory Comput. 2005, 1, 1128-1132; GDMA 2.2.04 at http://www-stone.ch.cam.ac.uk/programs.html). Those were obtained from the wavefunctions calculated at the MP2(full)/aug-cc-pVTZ level of theory using the Gaussian program package. Grid-based quadrature for partitioning the contributions to the charge density from diffuse basis functions (via the keyword "SWITCH 4.0") was used. The point charges of $-0.72 \mathrm{e}$ $(\mathrm{O})$ and $0.36 \mathrm{e}(\mathrm{H})$ were obtained by the fit to the electrostatic potential (at MP2(full)/aug-cc-pVTZ level of theory; Merz-Kollman sampling scheme with ca. 6000 points per atom). The dipole-dipole interactions were calculated as described in the main text. 
Table S4: Energy decomposition analysis for the linear water dimer (in

\begin{tabular}{cccc}
\multicolumn{4}{c}{$\mathrm{kcal} / \mathrm{mol})$} \\
\hline SCF $^{a}$ & $\mathrm{SCF}+D I S P^{b}$ & $\mathrm{SAPT}^{b}$ CCSD $)^{c}$ \\
\hline$E_{\text {elst }}$ & -7.5 & -7.12 & -8.23 \\
$E_{\text {exch }}$ & 4.3 & 4.90 & 8.48 \\
$E_{\text {ind }}$ & $-0.5(\mathrm{PL}) /-1.8(\mathrm{CT})$ & -1.63 & -2.32 \\
$E_{\text {disp }}$ & - & -1.54 & -2.45 \\
$E_{\text {tot }}$ & -5.5 & -5.39 & -4.52 \\
\hline
\end{tabular}

${ }^{a}$ Umeyama \& Morokuma, J. Am. Chem. Soc. 1977, 99, 1316 (HF/6-31G**)

${ }^{b}$ Jeziorski \& van Hemert, Mol. Phys. 1976, 31, 713 (SCF, variation-perturbation treatment)

${ }^{c}$ Korona, Mol. Phys. 2013, 111, 3705 (SAPT(CCSD)/aug-cc-pVTZ)

Table S5: Energy decomposition analysis for adenine-thymine and guanine-cytosine dimers (in $\mathrm{kcal} / \mathrm{mol})^{a}$

\begin{tabular}{ccc} 
& \multicolumn{3}{c}{ guanine-cytosine dimers $($ in $\mathrm{kcal} / \mathrm{mol})$} \\
\hline$E_{\text {elst }}$ & $-29.15^{b}\left(-30.06^{c}\right)$ & guanine-cytosine \\
$E_{\text {exch }}$ & $37.43(33.08)$ & $-45.56(-47.92)$ \\
$E_{\text {ind }}$ & -12.72 & $50.62(44.42)$ \\
$E_{\text {disp }}$ & -11.09 & -20.86 \\
$E_{\text {tot }}$ & -15.54 & -14.41 \\
\hline
\end{tabular}

${ }^{a}$ Heßelmann et al. J. Am. Chem. Soc. 2006, 128, 11730

${ }^{b} \mathrm{SAPT}(\mathrm{DFT}): \mathrm{LPBE0AC} /$ aug-cc-pVQZ

${ }^{c} \mathrm{SAPT}(\mathrm{HF}) /$ aug-cc-pVQZ (in parentheses) 